\title{
Surviving in steep terrain: a lab-to-field assessment of locomotor costs for wild mountain lions (Puma concolor)
}

\author{
Carolyn E. Dunford ${ }^{1}$ (D, Nikki J. Marks ${ }^{1}$, Christopher C. Wilmers², Caleb M. Bryce ${ }^{3}$, Barry Nickel ${ }^{2}$, Lisa L. Wolfe ${ }^{4}$, \\ D. Michael Scantlebury ${ }^{1^{*}}$ (D) and Terrie M. Williams ${ }^{5^{*}}$
}

\begin{abstract}
Background: Under current scenarios of climate change and habitat loss, many wild animals, especially large predators, are moving into novel energetically challenging environments. Consequently, changes in terrain associated with such moves may heighten energetic costs and effect the decline of populations in new localities.

Methods: To examine locomotor costs of a large carnivorous mammal moving in mountainous habitats, the oxygen consumption of captive pumas (Puma concolor) was measured during treadmill locomotion on level and incline $\left(6.8^{\circ}\right)$ surfaces. These data were used to predict energetic costs of locomotor behaviours of free-ranging pumas equipped with GPS/accelerometer collars in California's Santa Cruz Mountains.
\end{abstract}

Results: Incline walking resulted in a $42.0 \% \pm 7.2$ SEM increase in the costs of transport compared to level performance. Pumas negotiated steep terrain by traversing across hillsides (mean hill incline $17.2^{\circ} \pm 0.3 \mathrm{SEM}$; mean path incline $7.3^{\circ} \pm 0.1 \mathrm{SEM}$ ). Pumas also walked more slowly up steeper paths, thereby minimizing the energetic impact of vertical terrains. Estimated daily energy expenditure (DEE) based on GPS-derived speeds of free-ranging pumas was $18.3 \mathrm{MJ}$ day $^{-1} \pm 0.2 \mathrm{SEM}$. Calculations show that a 20 degree increase in mean steepness of the terrain would increase puma DEE by less than $1 \%$ as they only spend a small proportion (10\%) of their day travelling. They also avoided elevated costs by utilizing slower speeds and shallower path angles.

Conclusions: While many factors influence survival in novel habitats, we illustrate the importance of behaviours which reduce locomotor costs when traversing new, energetically challenging environments, and demonstrate that these behaviours are utilised by pumas in the wild.

Keywords: Energy expenditure, Energetics, Oxygen consumption, Indirect calorimetry, Locomotion, Incline, Carnivore, Accelerometer, Global change

\section{Background}

The ability of individual animals and animal populations to survive depends, in part, on their ability to balance

\footnotetext{
*Correspondence: m.scantlebury@qub.ac.uk; williams@biology.ucsc.edu 'School of Biological Sciences, Institute of Global Food Security, Queen's University of Belfast, 19 Chlorine Gardens, Belfast BT9 5DL, Northern Ireland ${ }^{5}$ Department of Ecology and Evolutionary Biology, Coastal Biology Building, 130 McAllister Way, University of California- Santa Cruz, Santa Cruz, CA 95060, USA

Full list of author information is available at the end of the article
}

energy expenditure with energy acquisition within a stochastic environment [1]. In recent years, the behavioural and physiological repertoire of large predatory mammals to maintain energy balance has been challenged by the magnitude and rapidity of environmental perturbations associated with climate change and habitat loss $[2,3]$. One of the most obvious and energetically costly responses necessary to meet the comparatively highresource demands of a carnivorous lifestyle [4], is

C C The Author(s). 2020 Open Access This article is licensed under a Creative Commons Attribution 4.0 International License, which permits use, sharing, adaptation, distribution and reproduction in any medium or format, as long as you give appropriate credit to the original author(s) and the source, provide a link to the Creative Commons licence, and indicate if changes were made. The images or other third party material in this article are included in the article's Creative Commons licence, unless indicated otherwise in a credit line to the material. If material is not included in the article's Creative Commons licence and your intended use is not permitted by statutory regulation or exceeds the permitted use, you will need to obtain permission directly from the copyright holder. To view a copy of this licence, visit http://creativecommons.org/licenses/by/4.0/ The Creative Commons Public Domain Dedication waiver (http://creativecommons.org/publicdomain/zero/1.0/) applies to the data made available in this article, unless otherwise stated in a credit line to the data. 
associated with an increase in distance moved in search of food, often requiring travel into and within new habitats [5].

Because locomotory activities often account for a large proportion of a mammal's daily energy expenditure (DEE) (see [6-8] for a discussion of transport costs), energy balance can be compromised in highly mobile carnivores as daily activity increases $[9,10]$. Changes in body orientation, gait, speed, and manoeuvring have been linked to environmental factors $[11,12]$ and subsequently to increased overall energy expenditure. It follows that locomotor responses that promote maximum energetic efficiency while minimizing the costs of transport when transiting though difficult terrains are considered beneficial [13].

Recently, there has been growing interest in using accelerometer-based technology to record simultaneous behavioural and energetic responses of animals transiting different habitats (for example $[10,14,15]$ ). To date, accelerometers have been used primarily to predict energetic costs associated with various body movements, changes of direction, and gaits [15-17]. By comparison, few studies have specifically used this technology to assess the energetic consequences of travelling in different terrains [18]. Higher energetic costs are presumed to occur in more challenging environments such as thick vegetation and sandy soils $[19,20]$. Similarly, locomotion on inclines can instigate an increase in energy expenditure compared to that incurred on level ground [21]. Consequently, large animals, such as elephants are suggested to avoid shallow incline slopes when travelling to minimise energetic costs [22-24]. Despite this, some mammals appear to select what would be considered energetically disadvantageous habitats (i.e., mountain ranges). Here the puma (Puma concolor) provides a unique opportunity to evaluate how energetic balance and locomotor efficiency can be maintained in a large predator that moves across a complex landscape.

Pumas frequent a wide range of habitats in North and South America [25], which includes mountainous, challenging terrains. This medium-sized (41-68 kg) felid has large home ranges (up to $723 \mathrm{~km}^{2}$ ) [26]. However, they are considered to be specialist 'stalk-and-pounce' predators $[26,27]$ and it has been suggested that pumas may be energetically constrained by an inability to increase energy demands due to their low aerobic scope [28]. This physiological limitation coupled with habitat loss and fragmentation will likely have an impact on the numbers and distribution of pumas as they are increasingly pressured to move into novel environments with unpredictable energetic demands [29]. If wild pumas are constrained by a low aerobic scope, pumas in steep terrains would have to travel slowly and on shallow inclines to avoid exceeding their lactate threshold [28, 30].
Alternatively, pumas could spend the majority of their time at rest in order to decrease the effect of terrain on overall energy expenditure and/or recover from high exercise performance levels.

To determine how locomotory strategies used by pumas may mitigate the potentially high costs of living in mountainous habitats, we investigated one obvious challenge; how steep terrain influences DEE. This was achieved by quantifying the energetic costs of walking on different gradients and at different speeds in the laboratory, and then monitoring how wild pumas living in the Santa Cruz Mountains (California) accrue or avoid these costs. For wild pumas, we assessed daily behaviours and assigned an energetic cost to each based on the laboratory measurements. The wild puma behaviour was identified from GPS and accelerometer data that were calibrated through observations of captive pumas, and the gradient and speed of their locomotion was assessed from the difference in altitude and distance between GPS points. Using this approach, we found that extraordinary metabolic demands due to steep terrains were circumvented by, 1) spending a low proportion of the day actively moving, 2) avoiding steep inclines by horizontally traversing hillsides, and 3) walking more slowly on inclines.

\section{Methods}

In a lab-to-field protocol, we used trained pumas to calibrate SMART collars (described below) that were subsequently deployed on wild counterparts in the Santa Cruz Mountains (CA). Details of the instrumentation and the development of behavioural and energetic signatures from accelerometers incorporated into the SMART collars have been reported previously [14], and are summarized here.

\section{Laboratory energetics \\ Animals}

Three adult pumas $(\mathrm{n}=$ two males, one female, body mass $=65.7 \pm 4.4 \mathrm{~kg}$ ), that originated as cubs from the wild, were hand-reared and trained using operant conditioning methods over 10 months to walk in a metabolic chamber mounted on a motorized treadmill. The animals lived in a natural habitat $(50 \mathrm{~m} \times 60 \mathrm{~m}$ pens) connected to an indoor facility (Foothills Wildlife Research Facility - Colorado Division of Parks and Wildlife, Fort Collins, CO, USA) and fed wild game carcass 2-3 times weekly and diced game meat daily when training. Energetic and kinematic tests were conducted during both winter and summer months. Ambient air temperature during the treadmill tests, $\mathrm{T}_{\text {air }}$, ranged from $11.1^{\circ} \mathrm{C}$ (winter) to $34.0^{\circ} \mathrm{C}$ (summer). 


\section{Open-flow respirometry}

Pumas rested and walked on a variable-speed, motorized treadmill (PAWWWS Treadmills, Carson, IA, USA) with a Plexiglas and steel framed metabolic chamber $(62$ $\mathrm{cm} \mathrm{W} \times 92 \mathrm{~cm} \mathrm{H} \times 200 \mathrm{~cm} \mathrm{~L}$ ) mounted on top. Pumas were post-absorptive following an overnight fast, and tested once per day. Each session began with a preexercise resting measurement on sedentary animals followed by steady-state walking or running at a single speed. Total time in the respirometer was approximately 20-30 min with each steady-state trial period lasting a minimum of $10 \mathrm{~min}$. Two pumas voluntarily walked on a level and a moderate incline $\left(6.8^{\circ}\right)$ surface at speeds up to $2 \mathrm{~m} \mathrm{~s}^{-1}$ and $1.1 \mathrm{~m} \mathrm{~s}^{-1}$, respectively. One puma was included in resting measurements only. Incline angle was selected to provide an additional energetic challenge to the pumas while ensuring that the animals could complete the $10 \mathrm{~min}$ test period.
The rate of oxygen consumption $\left(\mathrm{VO}_{2}\right)$ was determined using the protocols of Williams et al. [14] (and see Supplementary Information). The rate of oxygen consumption $\dot{V} \mathrm{O}_{2}$ was determined using the protocols of Williams et al. [14] (see also Supplementary Information). The cost of transport (COT) was calculated by dividing $\mathrm{VO}_{2}$ by treadmill speed during each test. Puma $\mathrm{VO}_{2}$ was calibrated with overall dynamic body acceleration (ODBA, see below) from the output of accelerometers on the SMART collar by Williams et al. [14].

\section{Behavioural signature library}

Accelerometer signals from the collars were related to specific behaviours by recording daily movements of collared, captive pumas. A running diary for each animal was recorded by observers with stopwatches and supplemented with video sequences (30 fps, Sony HDR-CX240, a

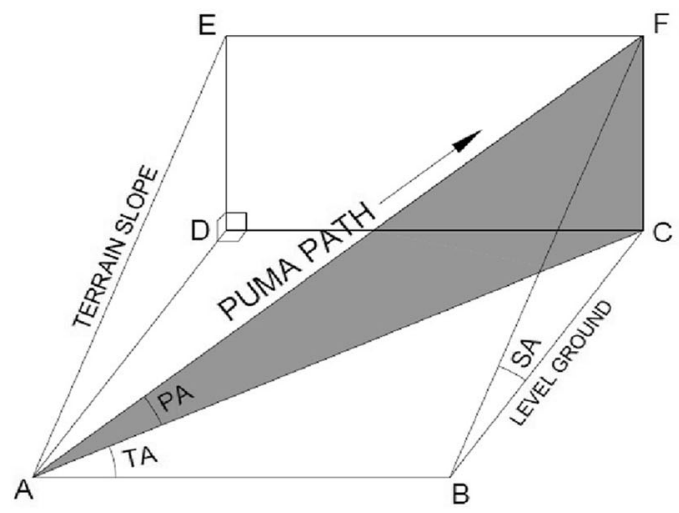

b

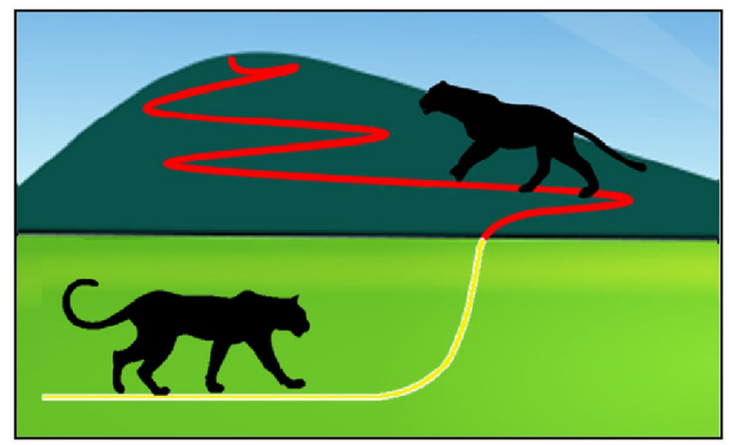

\begin{tabular}{|l|l|}
\hline Code & Measurement \\
\hline$A C$ & GPS distance \\
\hline$A F$ & Path distance \\
\hline$C F$ & Elevation gain \\
\hline$\angle F A C$ & Path angle (PA) \\
\hline$\angle F B C$ & Topographical slope angle (SA) \\
\hline$\angle C A B$ & Traverse angle (TA) \\
\hline
\end{tabular}

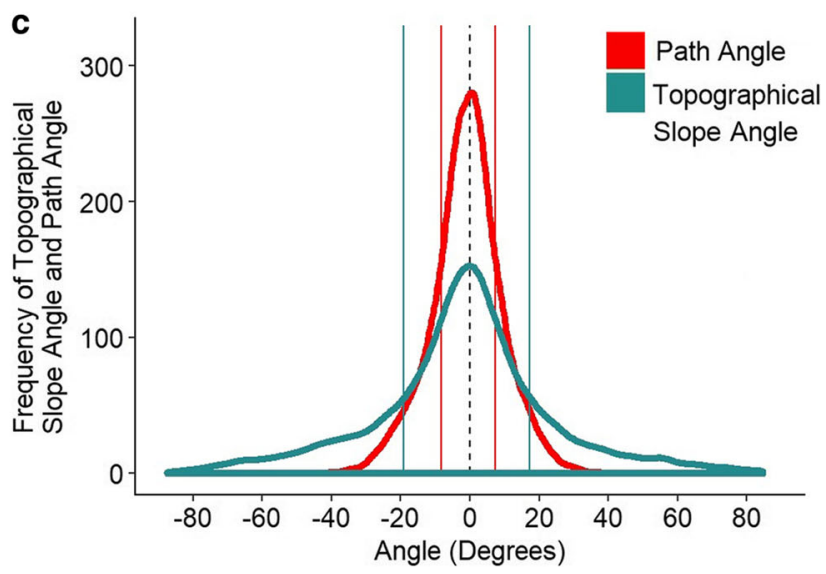

Fig. 1 a "Cheese wedge" model of slope climbed by pumas. Topographical slope angle (SA) describes the steepness of the hill the puma is standing on. Path angle (PA) is the steepness of the path taken by the puma as it walks up the hill (PA $\leq$ SA). Traverse angle (TA) is the horizontal angle that the puma walks up the hill. The horizontal GPS distance travelled is the distance between A and C. The path distance the puma travelled, is the distance between points $A$ and $F$. The elevation gain is the distance between C and F. b A schematic showing proposed travelling methods by pumas for different slopes. The yellow path is locomotion on the level ground (direct routes) and the red path is traversing inclining terrain. c Frequency of topographical slope angles encountered (green), and path angles chosen by pumas (red). Mean (solid line) shown for incline and decline for each. The dashed line is zero degrees 
Sony Corp., USA, described in detail in ref. [14]) (see also Supplementary Information). Behaviour categories that were considered to be important for the wild pumas were locomotion, non-mobile activities (e.g. eating and grooming), and resting. These are a subset of the behaviour categories detailed in Williams et al. [14], in which the focal behaviours were uniquely identified. These same categories were then used to create a decision tree which also incorporated GPS-derived speed to confirm when the pumas were travelling (Supplementary Fig. 1).

\section{Field research}

\section{Study animals and site}

Four wild adult male pumas were fitted with a SMART neck collar consisting of an integrated GPS/accelerometer logger (model GPS Plus, Vectronics Aerospace, Germany; total collar mass $=480 \mathrm{~g}$ ). The animals were captured using either trailing hounds or cage traps, as part of a larger study on puma ecology. Pumas were anesthetized with Telazol $^{\circ}$ (Fort Dodge Laboratories, Fort Dodge, IA, USA), sexed, weighed, measured for length, and fitted with an identifying ear tag and collar. Males were selected because they are more mobile than females [31]. GPS loggers recorded one fix every five minutes and tri-axial accelerometers recorded at $32 \mathrm{~Hz}$ [32] for the duration of the study period. Pumas were monitored for approximately 2 months each $(58.25 \pm 2.56$ days $)$ in 2016; two starting in May, one in October, and one in December (see Supplementary Table 2). The study area, the Santa Cruz Mountains (California, USA), ranges from sea level to $1155 \mathrm{~m}$ elevation and has a Mediterranean climate with a diverse landscape of urban developments as well as undisturbed native vegetation (see [31]).

\section{Landscape and path descriptions}

GPS location data were used to provide information on the distances and speed of travel, and the elevation change corresponding with that period. Puma home ranges were calculated as $95 \%$ minimum convex polygons (MCP) using a custom program based on the Python programming language (v. 2.7.9; Python Software Foundation, Wilmington, DE, USA). Elevation change was determined as the difference in elevation between sequential five-minute fixes where elevation was extracted for each location from an underlying digital elevation model (United States Geological Survey 2011). Similarly, horizontal distances travelled were determined as the distance between successive five-minute geolocation fixes. GPS-derived speed was calculated from distance travelled and time, and accelerometer-derived speed (in $\mathrm{m} \mathrm{s}^{-1}$ ) was calculated from ODBA (in $g$ ) using the relationship

$$
\text { Speed }=5.32 * O D B A-0.42
$$

from Williams et al. [14]. During the observation period, pumas travelled through the mountainous landscape and climbed up and down varied slopes (Figs. 1 and 2). They could either travel up the slope of a hill directly, walking up the steepest possible angle of the hill, or they could traverse along the side of a hill at a shallower angle and thereby travel a longer distance. Pumas were assumed to travel in a straight line between sequential GPS recordings, and hence distances recorded were minimal distances travelled. To distinguish between the different paths chosen by pumas relative to the steepness of the hill, we used the terms, 1) GPS distance, 2) path distance, 3) elevation gain, 4) topographical slope angle, 5) path angle, and 6) traverse angle as defined by the angular deviation between compass headings of the path and the compass direction of the topographical slope (i.e. topographical aspect, Fig. 1a). Path distances travelled, and path angles taken were calculated geometrically. Topographical slope angles and path angles were either positive (inclining) or negative (declining); steep angles become more vertical, reaching $+90^{\circ}$ (inclining) or $-90^{\circ}$ (declining) and shallow angles are close to $0^{\circ}$. Traverse angles were determined so that values approaching $0^{\circ}$ represented travel perpendicular to topographical slope (i.e. cross-slope travel), and $90^{\circ}$ travel parallel to the direction of the slope (Fig. 1a).

\section{Behaviour identification}

For each five-minute period, puma behaviour was classified into one of five categories, 1) resting, 2) non-mobile behaviours (e.g. feeding or grooming), 3) locomotion on an incline, 4) locomotion on a decline, and 5) unknown. Categorization was based on the GPS-derived speed and gradient that the animal was travelling and the relative activity, as recorded by the tri-axial accelerometer, in $g$ $\left(\mathrm{m} \mathrm{s}^{-2}\right)$ (see Supplementary Information).

\section{Calculating $\mathrm{VO}_{2}$ for pumas in the wild}

The relationship between $\dot{V} \mathrm{O}_{2}$ while walking and surface gradient is described as linear and positive at slow speeds and shallow gradients [21, 33, 34]. Therefore, we assumed that the $\mathrm{VO}_{2}$ of wild pumas increased linearly with the gradient of the angle they were climbing. To calculate puma $V \mathrm{O}_{2}$ for inclines in the field, the difference between mean $\dot{V} \mathrm{O}_{2}$ on the level and on the $6.84^{\circ}$ treadmill incline was calculated for each of the speeds where data for both were obtained $\left(0.56-1.11 \mathrm{~m} \mathrm{~s}^{-1}\right)$. The difference in $\dot{V} \mathrm{O}_{2}$ was then divided by $6.8^{\circ}$ (the treadmill angle) to give the $\mathrm{VO}_{2}$ increase per one degree of incline. Hence, the $\dot{V} \mathrm{O}_{2}$ values of wild pumas traversing various mountain slopes were calculated as the $\mathrm{VO}_{2}$ incurred when travelling on level ground plus the 

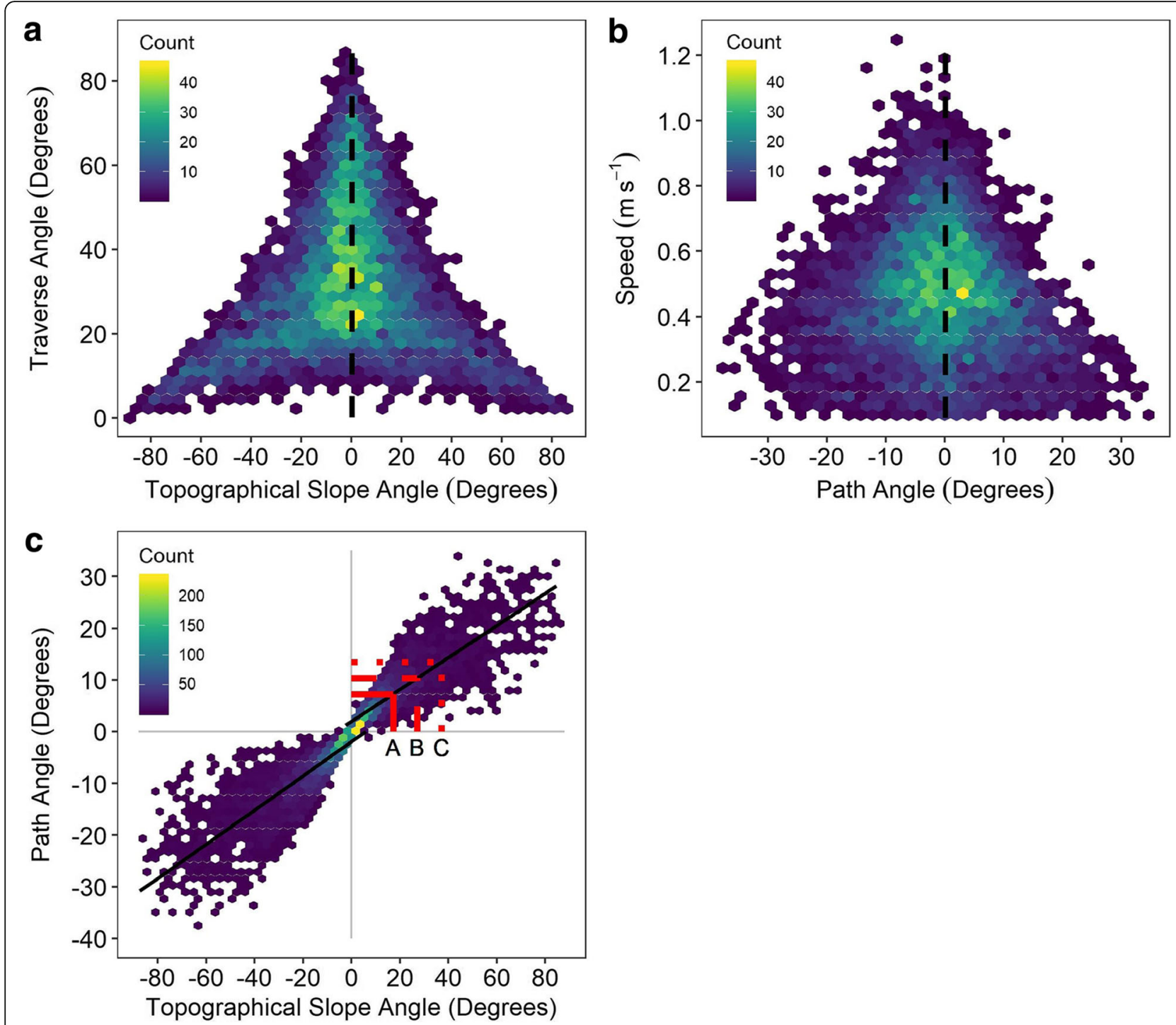

Fig. 2 Density plots of (a) traverse angles in relation to topographical slope angle and (b) speed in relation to path angle and speed in relation to path angle. Positive path angles signify pumas walking on inclines. Negative path angles signify pumas walking on declines. The dashed line indicates level walking $\left(0^{\circ}\right)$. Colour scales represents the density of points (yellow = high density, blue = low density according to the 'Count' scale). (c): Density plot for topographical slope angle of the terrain in relation to path angle (selected incline by pumas). Least square regression lines are shown separately for incline and decline data (based on Eqs. 6 and 7). For incline data, the solid line A represents mean topographical slope angle and mean path angle. Dashed line B and dotted line $\mathrm{C}$ represent an extrapolation from the incline regression using a mean $10^{\circ}$ or $20^{\circ}$ increase in the topographical slope angle. The mean path angle would increase to $10.4^{\circ}$ and $13.5^{\circ}$ respectively. The colour represents density of points as described above

additional $\mathrm{VO}_{2}$ incurred as a result of travelling on a particular incline. By extrapolation, we calculated the $\mathrm{VO}_{2}$ of pumas walking on any angle at any speed (see Eq. 9) which was verified by comparison with other felids (see Supplementary Information).

In this study, declining path angles were assumed to result in a $\dot{V} \mathrm{O}_{2}$ cost similar to that of level locomotion. This is based on data reported by Raab et al. [35] in which the $\mathrm{VO}_{2}$ values of dogs walking on the level or angles down to $-20.4^{\circ}$ were not significantly different, and on the results of recent studies that have investigated the energetic cost of decline walking for other species [21].

The $\mathrm{VO}_{2}$ (in $\mathrm{mlO}_{2} \mathrm{~kg}^{-1} \mathrm{~min}^{-1}$ ) of wild pumas engaged in non-mobile activities (i.e., feeding, grooming) was calculated using ODBA (in $g$ ) as described in detail in Williams et al. [14] Eq. 2 where

$$
\dot{V}_{2}=58.42 * O D B A+3.52
$$

$\mathrm{VO}_{2}$ was converted to a whole-body field energetic cost 
(in kilojoules) by multiplying by $20.1 \mathrm{~J} \mathrm{ml}^{-1}$ and the puma's mass (in kg) [36].

\section{Statistics}

Analyses were conducted using $\mathrm{R}$ (version 3.4.0, $\mathrm{R}$ core team 2014), with a statistical significance level of $p<$ 0.05 used. The results, unless otherwise indicated, are expressed as mean \pm 1 standard error. General linear mixed (GLM) models were used to examine interactions between speed and treadmill angle on $\mathrm{VO}_{2}$ (Supplementary Table 1). Each measurement on the treadmill was treated as an individual data point. GLM models were also used to determine the explanatory variables which best predicted traverse angle and speed during locomotion of wild puma on inclines. Likewise, GLM models were used to examine the interactions between topographical slope angle and traverse angle to explain path angle. Puma ID was included as a random factor. Fvalues were calculated using ANOVAs. Residuals of each model were examined for normality using QQplots.

\section{Results}

\section{Oxygen consumption $\left(\mathrm{V}_{2}\right)$ during level and incline walking}

The mean $\dot{V O}_{2}$ of pumas at rest was $8.22 \pm 1.09$ $\mathrm{mlO}_{2} \mathrm{~kg}^{-1} \mathrm{~min}^{-1}$, and increased with treadmill speed (in $\mathrm{m} \mathrm{s}^{-1}$ ) (Fig. 3a) where the least-squares fitted regression

$$
\dot{V} \mathrm{O}_{2}=10.99 * \text { speed }+8.15
$$

explained $94.9 \%$ of the variation in $\dot{V} \mathrm{O}_{2}\left(\mathrm{~F}_{1,18}=335.8\right.$, $p<0.001$ ) (see also [14] Eq. 1). When pumas were walking at a $6.8^{\circ}$ incline, $\dot{V} \mathrm{O}_{2}$ also increased with higher treadmill speeds, at almost twice the rate. In this case, the resulting least-squares fitted regression

$$
\dot{V} \mathrm{O}_{2}=21.29 * \text { speed }+8.83
$$

explained $90.6 \%$ of the variation in $\dot{V} \mathrm{O}_{2} \quad\left(\mathrm{~F}_{1,14}=134.2\right.$, $p<0.001)$. There was a significant interaction between speed and incline on $\dot{V O}_{2} \quad\left(\chi^{2}=59.16, p<0.001\right)$ such that $\dot{V} \mathrm{O}_{2}$ increased with speed at a faster rate when pumas were walking on an incline compared with when they were walking on the level. The highest $\dot{V} \mathrm{O}_{2}$ value recorded was $38.27 \mathrm{mlO}_{2} \mathrm{~kg}^{-1} \mathrm{~min}^{-1}$ when a puma was walking at its maximum voluntary speed $\left(1.1 \mathrm{~m} \mathrm{~s}^{-1}\right)$ on the inclined treadmill.

The COT decreased with faster speeds $\left(\mathrm{F}_{1,1}=124.87\right.$, $p<0.001)$, but increased with incline $\left(\mathrm{F}_{1,1}=171.37, p<\right.$ $0.001)$. There was no significant interaction between speed and incline on COT $\left(\mathrm{F}_{1,1}=0.03, p=0.85\right)$; COT decreased with speed at a similar rate during incline and level locomotion. The COT of pumas on the incline $\left(0.15 \pm 0.02 \mathrm{mlO}_{2} \mathrm{~kg}^{-1} \mathrm{~m}^{-1}\right)$ was $41.9 \pm 7.20 \%$ greater than on the level.

\section{Wild puma behaviour}

In total, 6037 five-minute windows of locomotory events were identified for the four pumas (mean per puma = $1509 \pm 396$ events, of which $715 \pm 219$ were incline events, and $795 \pm 182$ were decline events) (Supplementary Table 2). The four wild pumas spent a mean time travelling of $134.52 \pm 8.30 \mathrm{~min}$ per day (9.3\% of day). During this time, pumas spent $66.6 \pm 4.6$ min per day (4.6\% of day) walking up inclining paths and $71.2 \pm 4.0$ min per day (5.0\% of day) walking down declining paths. Non-mobile activities such as feeding and grooming accounted for $398.8 \pm 8.7 \mathrm{~min}$ per day (27.7\% of day). The pumas spent $851.4 \pm 13.3 \mathrm{~min}$ per day (59.1\% of day) resting. Unknown behaviours accounted for $37.7 \pm 3.0 \mathrm{~min}$ per day $(2.7 \pm$ $0.2 \%$ of day). Individual pumas showed different ranging behaviours and home ranges (Supplementary Figure 2 and Supplementary Table 3).

Locomotory events identified for the four individuals indicated that the animals used a wide variety of terrains. The mean topographical slope angle encountered while climbing uphill was $17.2 \pm 0.3^{\circ}$ (median $=11.0^{\circ}$, maximum $\left.=84.6^{\circ}\right)$. This compares with a mean decline topographical angle of $-19.1 \pm 0.3^{\circ}$ (median $=-12.8^{\circ}$, minimum $=-87.5^{\circ}$ ) encountered while going downhill (Fig. 1c). During the study, pumas never climbed directly up steep slopes. Instead, they climbed steep slopes by traversing and choosing shallower path angles (mean inclining path angle $7.3 \pm 0.1^{\circ}$, median $=5.5^{\circ}$ ). Pumas also avoided walking down steeply declining slopes directly (mean declining path angle $-8.3 \pm 0.1^{\circ}$, median $=-6.3^{\circ}$ ). The maximum path angle for a climbing puma was $34.3^{\circ}$; the minimum for a descending puma was $-37.0^{\circ}$ (Fig. 1c).

During incline locomotion, there was a significant negative effect of topographical slope angle on traverse angle $\left(\chi^{2}=1603.8, p<0.001\right)$. Thus, as the topographical slope angle increased, the traverse angle and the path angle of the puma decreased (Fig. 2). Furthermore, there was a significant interaction between topographical slope angle and traverse angle on path angle $\left(\chi^{2}=8182.4, p<0.001\right)$. As pumas encountered progressively steeper slopes, they climbed at progressively shallower angles relative to the steepness of the slope. This strategy enabled pumas to avoid directly climbing up steep slopes (Fig. 1b). Consequently, $95 \%$ of path angles that pumas climbed were shallower than $19.74^{\circ}$. Last, there was a significant negative effect of path angle on the speed at which pumas travelled $\left(\chi^{2}=521.4, p<0.001\right)$, such that pumas travelled along shallower path angles at faster speeds and along steeper path angles at slower speeds (Fig. 2b).

Pumas also altered their behaviour during decline locomotion; as the topographical slope angle became more steeply downhill, traverse angle decreased (Fig. 2a). There was a significant positive effect of topographical slope angle on traverse angle $\left(\chi^{2}=1879.4, p<0.001\right)$ as well as a 


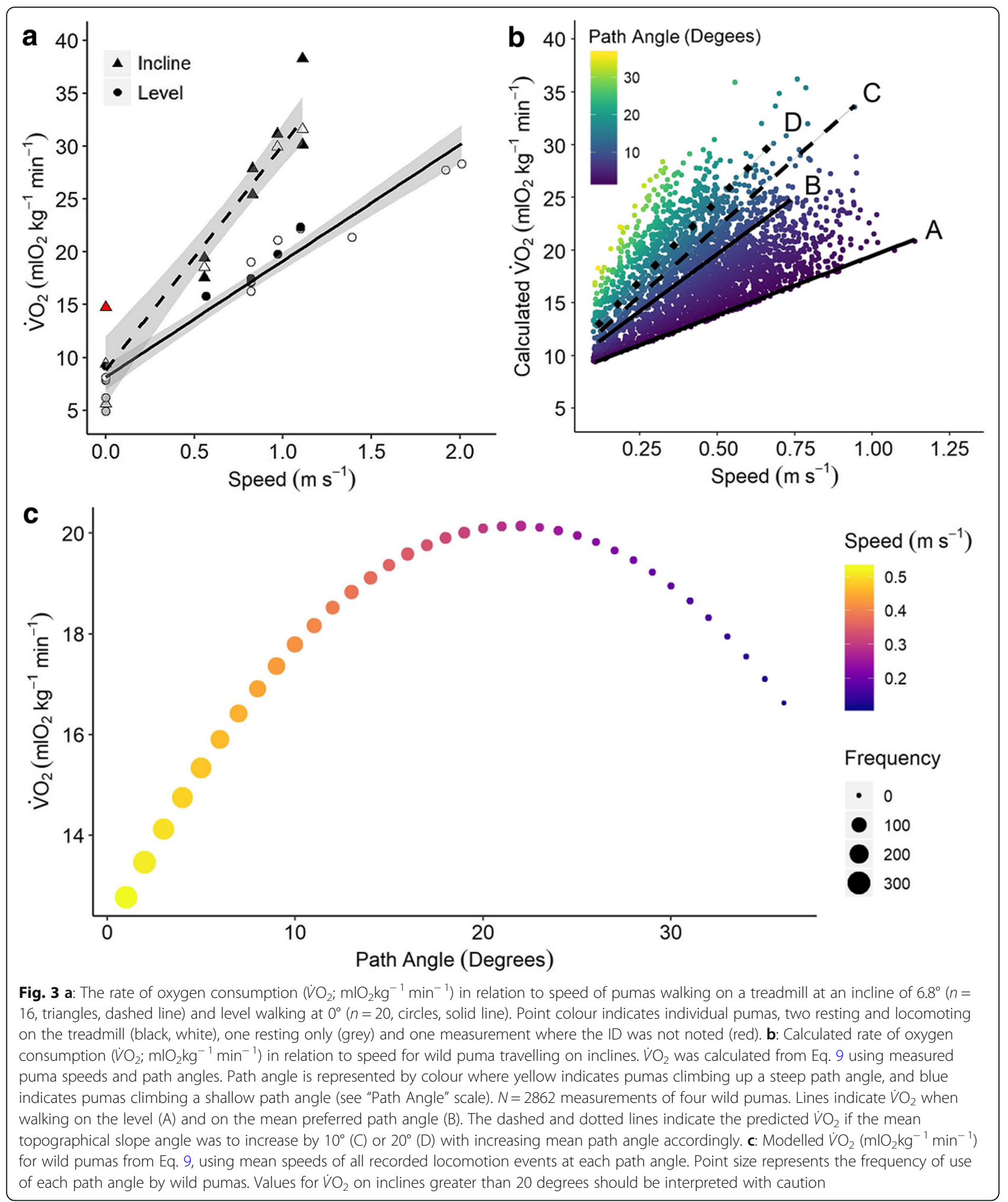

significant interaction between topographical slope angle and traverse angle on declining path angle $\left(\chi^{2}=7489.5\right.$, $p<0.001)$. These findings suggest that as pumas encountered progressively steeper downhill gradients, they decreased the path angle steepness, thereby avoiding directly climbing down steep slopes. The speed of descent was affected, leading to a significant decrease in speed as declining path angle increased $\left(\chi^{2}=230.41, p<0.001\right)$. Thus, pumas travelled 
along shallower path angles at faster speeds and along steeper downhill path angles at slower speeds (Fig. 2b).

There was a positive relationship between path angle and topographical slope angle (Fig. 2c) as described by the least-squares regression

$$
\begin{aligned}
\text { Path Angle (degrees) } & \\
= & 0.38 * \text { Topographical Slope Angle(degrees) } \\
& -0.164
\end{aligned}
$$

This regression explained $87.4 \%$ of the variation in path angles $\left(\mathrm{F}_{1,6032}=41,949.4, p<0.001\right)$ when including both inclining and declining locomotion. There were, however, differences in the energetic cost of inclining and declining locomotion, and we cannot assume that pumas use the same path angles when travelling up and down the terrain. In view of this, the regressions are presented separately below. When pumas were travelling uphill only, the least-squares regression

$$
\begin{aligned}
& \text { Inclining Path Angle (degrees) } \\
& =0.31 * \text { Topographical Slope Angle(degrees) } \\
& \quad+1.98
\end{aligned}
$$

explained $74.3 \%$ of the variation in path angle $\left(\mathrm{F}_{1,2857}=\right.$ 8303.1, $p<0.001)$. Similarly, when pumas were travelling downhill only, the least-squares regression

$$
\begin{aligned}
& \text { Declining Path Angle (degrees) } \\
& \quad=0.33 * \text { Topographical Slope Angle(degrees) }-1.94
\end{aligned}
$$

explained $75.1 \%$ of the variation in path angle $\left(\mathrm{F}_{1,3170}=\right.$ 9735.1, $p<0.001)$. As might be expected due to increased options, there was more variation in the path angles chosen by pumas travelling on steeper inclines and declines. The range of path angles used was greatest at the topographical angles of -60 degrees and 50 degrees (grouped by 10 degrees; Fig. 2c).

\section{Energy expenditure of wild pumas}

In the wild, pumas encountered many different slope angles and climbed up and down many different path angles. For every degree of path angle incline above level travel, the additional increase in $\dot{V} \mathrm{O}_{2}$ with speed $\left(\mathrm{m} . \mathrm{s}^{-1}\right)$ was

$$
\dot{\mathrm{VO}}_{2} \text { cost per } 1 \text { degree incline }=(1.47 * \text { speed })+0.088
$$

$\left(n=4, r^{2}=99.98\right)$. Based on this, the energetic cost of incline travel can be determined as: the regression for level $\dot{V} \mathrm{O}_{2}$ (Fig. 3a) plus the additional $\mathrm{VO}_{2}$ cost per degree of incline multiplied by the path angle:

$$
\begin{aligned}
\mathrm{VO}_{2}\left(\mathrm{mlO}_{2} \mathrm{~kg}^{-1} \mathrm{~min}^{-1}\right) & =(8.15+10.99 * \text { speed }) \\
& +(\text { path angle } *(1.47 * \text { speed }+0.088))
\end{aligned}
$$

where path angle is in degrees and speed is in $\mathrm{m} \mathrm{s}^{-1}$. Eq. 9 can be used to calculate the $V \mathrm{O}_{2}$ of wild pumas during incline locomotion using GPS-derived speed and path angles. This determines $\dot{V} \mathrm{O}_{2}$ costs incurred across the range of speeds and path angles utilised by pumas (Fig. $3 b)$.

We found that the maximum $\mathrm{VO}_{2}$ during locomotion by a wild puma was $34.86 \mathrm{mlO}_{2} \mathrm{~kg}^{-1} \mathrm{~min}^{-1}$, which occurred at a path angle of $16.4^{\circ}$ and at a speed of $0.76 \mathrm{~m} \mathrm{~s}^{-1}$. The mean field $\dot{V} \mathrm{O}_{2}$ on inclines was $17.90 \pm 0.08 \mathrm{mlO}_{2} \mathrm{~kg}^{-1} \mathrm{~min}^{-1}$, and the mean field $\dot{V} \mathrm{O}_{2}$ during declining locomotion was $14.08 \pm 0.06$ $\mathrm{mlO}_{2} \mathrm{~kg}^{-1} \mathrm{~min}^{-1}$ (Table 1).

DEE of the pumas depended on the behaviours exhibited on any given day. The mean DEE for the four wild pumas was $18.29 \pm 0.15 \mathrm{MJ}^{d_{a y}}{ }^{-1}$ (see Table 1 and calculations in Supplementary Information). Most of their time and energy was spent resting and on non-mobile behaviours (Supplementary Figure 3). Locomotion accounted for $13.97 \%$ of puma DEE, with incline travelling accounting for approximately half $(7.58 \%)$ of this but only taking $4.65 \%$ of the total time. There were differences in the mean $\dot{V} \mathrm{O}_{2}$ of distinct behaviours (Table 1 ), leading to differences between time spent on the behaviour and the cost (Supplementary Figure 3); pumas spent more time on low-cost behaviours rather than costly activities. The calculated proportion of DEE used for locomotion increased to $24.64 \%$ when using accelerometer, rather than GPS-derived speed (Table 1).

\section{Discussion}

Energy landscape theory posits that animals generally avoid going through areas that pose a high energetic cost, and instead use a 'path of least resistance' strategy $[13,37]$. As inhabitants of American deserts, the tropical flats of the Florida Everglades as well as the mountains of North and South America, pumas seem particularly adept at challenging this energetic optimization theory by living in a wide variety of habitats often considered as energetically demanding [13, 38]. This versatility, and the fact that approximately one third of existing puma habitat on private land in California will be lost by 2030 due to increased urban sprawl [39], makes this felid a particularly relevant animal model for investigating strategies for surviving in difficult landscapes. Here we find that several key behavioural modifications may enable pumas to circumvent some of the anticipated energetic costs of inhabiting steep terrains. 
Table 1 Mean \pm SEM rate of oxygen consumption $\left(\mathrm{VO}_{2}\right)$ and mean daily energy expenditure (DEE) of pumas in the Santa Cruz Mountains, calculated for daily behaviours. GPS-derived and accelerometery-derived speeds are compared. See Supplementary Table 4 for equations used to calculate energy costs. ${ }^{\prime * \prime}$ indicates that the values are the same for GPS- and accelerometery-derived $\mathrm{VO}_{2}$ since these parameters are not based on speed

\begin{tabular}{|c|c|c|c|c|c|c|}
\hline \multirow[t]{2}{*}{ Behaviour } & \multicolumn{3}{|l|}{ GPS - derived speed } & \multicolumn{3}{|c|}{ Accelerometery - derived speed } \\
\hline & $\mathrm{VO}_{2}\left(\mathrm{mlO}_{2} \mathrm{~kg}^{-1} \mathrm{~min}^{-1}\right)$ & $\mathbf{V O}_{2}\left(\mathrm{mlO}_{2} \mathrm{~kg}^{-1} \mathrm{day}^{-1}\right)$ & Energy (MJ day ${ }^{-1}$ ) & $\overline{V_{2}}\left(\mathrm{mlO}_{2} \mathrm{~kg}^{-1} \mathrm{~min}^{-1}\right)$ & $\mathbf{V O}_{2}\left(\mathrm{mlO}_{2} \mathrm{~kg}^{-1}\right.$ day $\left.^{-1}\right)$ & Energy (MJ day $\left.{ }^{-1}\right)$ \\
\hline Decline Locomotion & $14.08 \pm 0.06$ & $1007 \pm 60.0$ & $1.18 \pm 0.07$ & $25.04 \pm 0.06$ & $1802 \pm 106.1$ & $2.11 \pm 0.12$ \\
\hline Incline Locomotion & $17.90 \pm 0.08$ & $1197 \pm 84.9$ & $1.40 \pm 0.10$ & $39.08 \pm 0.28$ & $2645 \pm 190.6$ & $3.09 \pm 0.22$ \\
\hline Non-mobile Activity & ${ }^{*} 15.23 \pm 0.05$ & ${ }^{*} 6190 \pm 128.0$ & $* 7.24 \pm 0.15$ & $* 15.23 \pm 0.05$ & ${ }^{*} 6190 \pm 128.0$ & $* 7.24 \pm 0.15$ \\
\hline Resting & *8.22 & $* 7101 \pm 103.8$ & $* 8.30 \pm 0.12$ & *8.22 & $* 7101 \pm 103.8$ & ${ }^{*} 8.30 \pm 0.12$ \\
\hline Unknown & *8.22 & $* 310 \pm 25.0$ & ${ }^{*} 0.36 \pm 0.03$ & *8.22 & $* 310 \pm 25.0$ & ${ }^{*} 0.36 \pm 0.03$ \\
\hline Total DEE & - & $15,640 \pm 126.8$ & $18.29 \pm 0.15$ & - & $17,661 \pm 242.0$ & $20.65 \pm 0.28$ \\
\hline
\end{tabular}

Locomotion on incline surfaces is known to be relatively costly for a wide variety of animals [21], including large mammals from dogs [35] and African lion cubs [33] to $440 \mathrm{~kg}$ thoroughbred racehorses [40]. As found in these previous studies, incline locomotion was more costly for pumas than level locomotion at comparable speeds with the difference in $\dot{V} \mathrm{O}_{2}$ for level and incline travel increasing progressively the faster the puma moved (Fig. 3a). Thus, the highest voluntary oxygen consumption rates on the treadmill (32-39 $\mathrm{mlO}_{2} \mathrm{~kg}^{-1}$ $\mathrm{min}^{-1}$ ) occurred at $2.0 \mathrm{~m} \mathrm{~s}^{-1}$ during level running, and at a lower speed of $1.1 \mathrm{~m} \mathrm{~s}^{-1}$ when on the incline.

Data from the SMART collars demonstrated how wild pumas avoided these excessive costs when traversing mountainous habitats. We found that pumas displayed a trade-off between speed, distance, and energy expenditure by modifying the chosen path of incline to affect the total cost of ascent (Figs. 1, 2 and 3). Through behavioural modifications, wild pumas could remain in an aerobic state during locomotor activity unless, for example, they were running at high speeds or on inclines during hunting. This is especially important for accommodating the relatively low aerobic scope of adult pumas (Fig. 3a [28]), where $V \mathrm{O}_{2}$ max is $\sim 5$ times resting levels rather than 610 times resting (at $49.2 \mathrm{mlO}_{2} \mathrm{~kg}^{-1} \mathrm{~min}^{-1}$ ) predicted for quadrupeds of a similar body mass [41].

$\dot{V} \mathrm{O}_{2}$ costs of pumas were minimised by utilising two behavioural adaptations. First, wild pumas did not climb directly up inclines. Instead, they traversed steep slopes and thereby decreased the actual path angle climbed (Fig. 2), which lowered the rate of increase of $\mathrm{VO}_{2}$ (Fig. 3b). Pumas decreased the mean path angle to $7.3^{\circ}$ from the mean topographical angle of $17.2^{\circ}$, and $95 \%$ of path angles were shallower than $19.74^{\circ}$. Second, wild pumas travelled slowly when they encountered steep slopes (Fig. 2b). The fastest speeds of $1.2 \mathrm{~m} \mathrm{~s}^{-1}$ were seen around a path angle of $0^{\circ}$, while the speed on the steepest $\left(>\right.$ c. $20^{\circ}$ ) path angles did not exceed $0.4 \mathrm{~m} \mathrm{~s}^{-1}$ (Fig. $2 \mathrm{~b})$. The speeds most commonly used by pumas were slower than previously reported; we recorded a high density of points around $0.4-0.6 \mathrm{~m} \mathrm{~s}^{-1}$ (Fig. 2b) for pumas in the wild. Previous estimates have been $1.1 \mathrm{~m}$ $\mathrm{s}^{-1}$ for level locomotion by pumas in an enclosure [14]. Slower speeds required a higher $\dot{V} \mathrm{O}_{2}$ per meter travelled (i.e. higher COT) and travelling at very slow speeds would not be energetically efficient, despite requiring the lowest $\dot{V} \mathrm{O}_{2}$ per minute, as it would take a long time to travel any distance. Indeed, regularly-used human footpaths follow this trend, where the footpath does not take the least time to reachthe destination, but instead maximises the efficiency of metabolic cost for human locomotion [42]. There is also a trade-off on steeper slopes where faster speeds are presumably not energetically sustainable, so pumas utilised slower speeds [21, 33, 35].

Similarly, pumas travelling on steeply descending slopes selected shallow traverse angles. These shallower path angles were also travelled at faster speeds than when they moved down steeply descending path angles (Fig. 2). Pumas travelling down very steep slopes could be at risk of stumbling if they travel quickly [18]. Increased energy might be required to slow their descent on steep declines [21], however these were rarely used by wild pumas. The 'bow tie' shape of selected path angles in relation to topographical slope angle (Fig. 2c) shows that both inclining and declining path angles had very similar regression slopes, indicating that similar path angles were used during both ascent and descent. This would presumably occur if pumas followed the same paths up and down hillsides. Indeed, the selected path angles of pumas do minimise the cost of travelling (Table 2). Our results indicate that the preferred path for pumas involved traversing around hills rather than travel over them, as observed in other large mammals [24].

Presumably, travelling a further distance when traversing a hillside to avoid steep inclines provides an energetic benefit to the puma. To test this, we created a simplistic theoretical model examining energy use at various speeds and slopes (Table 2). Based on simple 
Table 2 Theoretical model for the rate of oxygen consumption $\left(\mathrm{VO}_{2}\right)$ based on a puma walking $100 \mathrm{~m}$ up a $30^{\circ}$ path angle. Paths compared include climbing straight up or traversing back and forth at path angles of 2, 7, or 15 degrees. $\mathrm{VO}_{2}$ is calculated per min and then totalled for how long it would take the puma to reach the end point based on the distance travelled and speed. 'Poor' energetic options are indicated due to the $\mathrm{VO}_{2}$ exceeding maximum aerobic capacity or time exceeding 20 min. 'Good' energetic options show the optimal locomotion speed and path angle, and 'moderate' options are also indicated, which are often used

\begin{tabular}{|c|c|c|c|c|c|c|}
\hline Speed $\left(\mathrm{m} \mathrm{s}^{-1}\right)$ & Path angle (degrees) & $\mathrm{VO}_{2}\left(\mathrm{mlO}_{2} \mathrm{~kg}^{-1} \mathrm{~min}^{-1}\right)$ & Distance $(\mathrm{m})$ & Time (min) & Total $\mathrm{O}_{2}\left(\mathrm{mlO}_{2} \mathrm{~kg}^{-1}\right)$ & Energetic viability \\
\hline 0.1 & 2.0 & 9.7 & 1432.7 & 238.8 & 2320.7 & Poor \\
\hline 0.1 & 7.0 & 10.9 & 410.3 & 68.4 & 744.9 & Poor \\
\hline 0.1 & 15.0 & 12.8 & 193.2 & 32.2 & 411.3 & Poor \\
\hline 0.1 & 30.0 & 16.3 & 100.0 & 16.7 & 271.7 & Moderate \\
\hline 0.5 & 2.0 & 15.3 & 1432.7 & 47.8 & 730.2 & Poor \\
\hline 0.5 & 7.0 & 19.4 & 410.3 & 13.7 & 265.4 & Moderate \\
\hline 0.5 & 15.0 & 26.0 & 193.2 & 6.4 & 167.4 & Good \\
\hline 0.5 & 30.0 & 38.3 & 100.0 & 3.3 & 127.8 & Poor \\
\hline 1.0 & 2.0 & 22.3 & 1432.7 & 23.9 & 531.4 & Poor \\
\hline 1.0 & 7.0 & 30.0 & 410.3 & 6.8 & 205.5 & Moderate \\
\hline 1.0 & 15.0 & 42.5 & 193.2 & 3.2 & 136.9 & Poor \\
\hline 1.0 & 30.0 & 65.9 & 100.0 & 1.7 & 109.8 & Poor \\
\hline
\end{tabular}

oxygen consumed, it initially appears that running up steep inclines at fast speeds would use less energy than traversing due to the short time it takes to reach the top. However, a key factor that must be considered in such calculations is the added cost of anaerobic metabolism when climbing quickly up steep inclines. Modelled rates of oxygen consumption exceeded measured puma $\dot{V O}_{2}$ max for speeds greater than $0.5 \mathrm{~m} \mathrm{~s}^{-1}$ when combined with the steepest path angles. Overall, the lowest calculated cost for climbing that did not exceed aerobic limits would occur at a modest speed of $0.5 \mathrm{~m} \mathrm{~s}^{-1}$ with a path angle of $15^{\circ}$, which was similar to the observed movements of wild pumas (Fig. 2). Pumas in the wild may travel at modest speeds and shallower path angles to conserve energy, and to avoid entering anaerobic metabolism. Not surprisingly, anaerobically supported movements occur rarely for wild pumas except during brief bouts of prey capture (Fig. 2b; $[14,26]$ ) and in this study, during high-intensity escapes from trailing hounds used for puma capture (Supplementary Information [26]). Pumas running up and down hills during these hound escape sequences far exceeded the maximal values of oxygen consumption observed during treadmill tests, and one puma expended $>3 \%$ of the mean puma DEE during a chase of less than 7 minutes (Supplementary Table 5). Thus, it is not surprising that short, steep uphill sprints are rare for wild pumas, despite the benefit of short travel time.

For wild pumas walking on inclines, there was a parabolic relationship between locomotion speed and duration which resulted in a trade-off between maintaining a low field COT and climbing incline terrains quickly (Fig. 3C). The lowest measured COT occurred during faster movements on the level treadmill; such paths and were commonly used by the cats in the wild. The steepest path angles also resulted in lower $\mathrm{VO}_{2}$ costs compared with moderate incline angles performed at relatively fast speeds (Fig. 3c). This was due to the slow speeds used during these steep climbs and were rarely used by pumas.

Using these energetic data, we found that the calculated daily energy expenditure (DEE) of wild pumas, 18.29 $\mathrm{MJ} \mathrm{day}^{-1}$, living in a mountainous habitat was similar to that reported by Wilmers et al. [15] for wild African leopards (Panthera pardus) of $20.0 \mathrm{MJ} \mathrm{day}^{-1}$. This could be a conservative estimate of puma DEE as they may have strayed from direct paths between the GPS coordinates or lost and gained altitude in this time. The accelerometer-derived speed would account for both of these but calibrations of ODBA on inclines would be needed to improve the accuracy of $\mathrm{VO}_{2}$ calculations using this method.

Puma DEE values are higher than the allometric prediction for mammals of their body mass [43] (predicted $\left.9.42 \pm 0.27 \mathrm{MJ} \mathrm{day}^{-1}, \mathrm{t}(4.3)=-13.53, p<0.001\right)$. There may be multiple reasons for this (see Supplementary Information). Although the mountainous landscape could lead to a high DEE despite behavioural strategies for minimizing energy costs, this figure is likely to be an overestimate, perhaps resulting from extrapolation of laboratory data and/or an elevated RMR due to preexercise anticipation and excitement [44]. Importantly, 
wild pumas spent less than $10 \%$ of the day locomoting and any incremental increase in energy expenditure due to inclines would increase the pumas' DEE. The comparatively high DEE of the pumas in this study is also interesting as large carnivores are expected to maintain low energy expenditure due to unpredictable food sources [45]. The strategies seen here of pumas using predominantly slow speeds and shallow path angles indicate that they may be constrained by a low aerobic scope [28]. Pumas may use these strategies to ensure they do not fatigue or exceed their lactate threshold in steep terrains, both of which would prolong recovery times following exercise.

We can predict the impact of an increase in mean topographical slope angle by utilising the regressions above and therefore estimate the pumas' DEE in steeper habitats. Using Eq. 6, one can infer that an increase in mean topographical slope angle by $10^{\circ}$ or $20^{\circ}$ would increase the mean path angle to $10.39^{\circ}$ or $13.48^{\circ}$ respectively. Assuming that the pumas would use the same speeds at these path angles as our study pumas, mean $\mathrm{VO}_{2}$ (based on Eq. 9) would increase to 19.94 and $20.98 \mathrm{mlO}_{2} \mathrm{~kg}^{-1} \mathrm{~min}^{-1}$ for $10^{\circ}$ and $20^{\circ}$ slope increases, respectively. For the $20^{\circ}$ slope angle, this resulted in a $13 \%$ increase in $\mathrm{VO}_{2}$ for incline locomotion resulting in an increase in daily locomotion energy costs from $1.40 \mathrm{MJ}$ to $1.58 \mathrm{MJ}$ per day; as a proportion of total DEE, the increase would be $<1.0 \%$. It is important to note that steeper terrains could also increase $\mathrm{VO}_{2}$ costs due to steeper declines; in this study we have assumed that decline costs are the same as for level locomotion, however, there are some cases where very steep declines are more costly than level locomotion [21, 46]. Another assumption of these calculations is that there is a linear relationship between incline and the rate of increase of $\mathrm{VO}_{2}$ with speed. This may not be the case when pumas are travelling on the steepest slopes [21] and, while measurements of puma $V \mathrm{O}_{2}$ on steeper treadmill inclines would have been interesting to collect, lack of these data does not detract significantly from our daily energy estimates because the wild pumas utilised steep slopes (above $20^{\circ}$ ) less than $5 \%$ of the time they were travelling. Nevertheless, $\dot{V} \mathrm{O}_{2}$ estimates for inclines much steeper than this should be interpreted with caution. Future models constructed from higher temporal resolution collars and 'dead reckoning' path reconstruction [47] may also help to further detail the energetic costs of steep terrain in relation to its associated impact on travel routes, path angles, distance, and the consequential behavioural choices made by pumas.

\section{Conclusion}

This study investigated a single environmental challenge for pumas - incline locomotion - that must be considered in the context of other associated environmental factors that can affect overall energetic costs [19, 20]. Incline avoidance behaviours indicate a 'path of least resistance' strategy used by the pumas to decrease locomotion costs that will have an advantage through energetic conservation [48] especially in complex, costly habitats [14, 49]. Development of energy landscape modelling for pumas and other large carnivores could benefit from taking aspects of terrain - such as the steepness of slope - into account when identifying key habitats for conservation.

\section{Supplementary information}

Supplementary information accompanies this paper at https://doi.org/10. 1186/s40462-020-00215-9.

\section{Additional file 1.}

Additional file 2. Supplementary information

\section{Abbreviations}

GPS: Global positioning system; DEE: Daily energy expenditure; $\dot{V}_{2}$ : Rate of oxygen consumption; COT: Cost of transport; ODBA: Overall dynamic body acceleration; MCP: Minimum convex polygons; GLM: General linear mixed model

\section{Acknowledgements}

C.E.D. was supported by a DfE Northern Ireland Studentship awarded to D.M.S. and N.J.M. as well as a travel grant from the Company of Biologists Limited (Charity no. 277992), JEBTF-161119, sponsored by The Journal of Experimental Biology. NSF grants 0963022 and 1255913 were awarded to C.C.W. and T.M.W. The authors wish to thank Georgia Cope for her assistance with geometric figures and Jennifer Weller for her assistance with statistics, as well as Paul Houghtaling for his assistance in collecting field data. Animal procedures were approved by the UCSC IACUC.

\section{Authors' contributions}

TMW conceived the study; TMW and CCW collected the data; CED and BN analysed the data; CED and DMS conceived the lab-to-field energetics models and analyses. LLW trained and handled captive puma. All authors contributed to writing the manuscript. The authors read and approved the final manuscript.

Availability of data and materials

All data on Dryad Puma data, Dryad, Dataset, https://doi.org/10.5061/dryad. $4 q r j 6 q 6 w$ [50].

\section{Ethics approval and consent to participate}

Work involving live pumas (captive and wild) was carried out under protocols that were reviewed and approved for adherence to ethical and research goals by the University of California- Santa Cruz Institutional Animal Care and Use Committee (IACUC), (Approved protocol numbers: Willt1901 and Wilmc1612).

\section{Consent for publication}

Not applicable.

\section{Competing interests}

The authors declare that they have no competing interests.

\section{Author details}

${ }^{1}$ School of Biological Sciences, Institute of Global Food Security, Queen's University of Belfast, 19 Chlorine Gardens, Belfast BT9 5DL, Northern Ireland. ${ }^{2}$ Center for Integrated Spatial Research, Environmental Studies Department, University of California- Santa Cruz, Santa Cruz, CA 95064, USA. ${ }^{3}$ Botswana Predator Conservation Trust, Maun, Botswana. ${ }^{4}$ Colorado Division of Parks and Wildlife, Wildlife Health Program, 4330 Laporte Avenue, Fort Collins, CO 80521-2153, USA. ${ }^{5}$ Department of Ecology and Evolutionary Biology, Coastal 
Biology Building, 130 McAllister Way, University of California- Santa Cruz, Santa Cruz, CA 95060, USA.

\section{Received: 6 March 2020 Accepted: 9 June 2020}

Published online: 08 August 2020

\section{References}

1. Sibly RM, Grimm V, Martin BT, Johnston AS, Kułakowska K, Topping CJ, et al. Representing the acquisition and use of energy by individuals in agentbased models of animal populations. Methods Ecol Evol. 2013;4(2):151-61.

2. Rode KD, Robbins CT, Nelson L, Amstrup SC. Can polar bears use terrestrial foods to offset lost ice based hunting opportunities? Front Ecol Environ. 2015;13(3):138-45.

3. Wang Y, Smith JA, Wilmers CC. Residential development alters behavior, movement, and energetics in an apex predator, the puma. PLoS One. 2017; 12(10):e0184687.

4. The energetics of foraging in large mammals: a comparison of marine and terrestrial predators. International Congress Series: Elsevier; 2004.

5. Ropert-Coudert $Y$, Wilson RP. Trends and perspectives in animal-attached remote sensing. Front Ecol Environ. 2005:3(8):437-44.

6. Cavagna GA, Heglund NC, Taylor CR. Mechanical work in terrestrial locomotion: two basic mechanisms for minimizing energy expenditure. Am J Phys. 1977;233(5):R243-61.

7. Taylor CR, Heglund NC, Maloiy GM. Energetics and mechanics of terrestrial locomotion. I. Metabolic energy consumption as a function of speed and body size in birds and mammals. J Exp Biol. 1982:97:1-21.

8. Karasov WH. Daily energy expenditure and the cost of activity in mammals. Am Zool. 1992;32(2):238-48.

9. Gorman ML, Mills MG, Raath JP, Speakman JR. High hunting costs make African wild dogs vulnerable to kleptoparasitism by hyaenas. Nature. 1998 391(6666):479

10. Pagano AM, Durner GM, Rode KD, Atwood TC, Atkinson SN, Peacock E, et al. High-energy, high-fat lifestyle challenges an Arctic apex predator, the polar bear. Science. 2018;359(6375):568-72.

11. Shepard EL, Wilson RP, Quintana F, Laich AG, Liebsch N, Albareda DA, et al. Identification of animal movement patterns using tri-axial accelerometery. Endanger Species Res. 2008;10(2.1):47-60.

12. Ropert-Coudert $Y$, Grémillet $D$, Ryan $P$, Kato A, Naito Y, Le Maho Y. Between air and water: the plunge dive of the cape gannet Morus capensis. Ibis. 2004; 146(2):281-90.

13. Shepard EL, Wilson RP, Rees WG, Grundy E, Lambertucci SA, Vosper SB. Energy landscapes shape animal movement ecology. Am Nat. 2013;182(3): 298-312.

14. Williams TM, Wolfe L, Davis T, Kendall T, Richter B, Wang $Y$, et al. Mammalian energetics. Instantaneous energetics of puma kills reveal advantage of felid sneak attacks. Science. 2014;346(6205):81-5.

15. Wilmers CC, Isbell LA, Suraci JP, Williams TM. Energetics-informed behavioral states reveal the drive to kill in African leopards. Ecosphere. 2017;8(6): e01850

16. Wilson RP, Griffiths IW, Mills MG, Carbone C, Wilson JW, Scantlebury DM. Mass enhances speed but diminishes turn capacity in terrestrial pursuit predators. elife. 2015;4:e06487.

17. Bryce CM, Williams TM. Comparative locomotor costs of domestic dogs reveal energetic economy of wolf-like breeds.J Exp Biol. 2017:220(Pt 2):312-21.

18. Birn-Jeffery AV, Highman TE. The scaling of uphill and downhill locomotion in legged animals. Integr Comp Biol. 2014;56(6):1159-72.

19. Lejeune TM, Willems PA, Heglund NC. Mechanics and energetics of human locomotion on sand. J Exp Biol. 1998;201(Pt 13):2071-80.

20. Pinnington $\mathrm{HC}$, Dawson $\mathrm{B}$. The energy cost of running on grass compared to soft dry beach sand. J Sci Med Sport. 2001;4(4):416-30.

21. Halsey LG, White CR. A different angle: comparative analyses of wholeanimal transport costs when running uphill. J Exp Biol. 2017;220(Pt 2):161-6.

22. Reichman OJ, Aitchison S. Mammal trails on mountain slopes: optimal paths in relation to slope angle and body weight. Am Soc Naturalists. 1981;117(3): $416-20$

23. Langman VA, Roberts TJ, Black J, Maloiy GM, Heglund NC, Weber JM, et al. Moving cheaply: energetics of walking in the African elephant. J Exp Biol. 1995;198(Pt 3):629-32.

24. Wall J, Douglas-Hamilton I, Vollrath F. Elephants avoid costly mountaineering. Curr Biol. 2006;16(14):R527-9.
25. Sunquist M, Sunquist F. Wild cats of the world. Chicago: University of Chicago Press; 2017

26. Bryce CM, Wilmers CC, Williams TM. Energetics and evasion dynamics of large predators and prey: pumas vs hounds. Peer J. 2017:5:e3701.

27. McNab BK. The standard energetics of mammalian carnivores: Felidae and Hyaenidae. Can J Zool. 2000;78(12):2227-39.

28. Williams TM, Bengtson P, Steller DL, Croll DA, Davis RW. The healthy heart: lessons from nature's elite athletes. Physiology (Bethesda). 2015;30(5):349-57.

29. Nielsen C, Thompson D, Kelly M, Lopez-Gonzalez C. Puma concolor. The IUCN red list of threatened species; 2015. p. 2015-4.

30. Williams TM, Fuiman LA, Horning M, Davis RW. The cost of foraging by a marine predator, the Weddell seal Leptonychotes weddellii: pricing by the stroke. J Exp Biol. 2004;207(Pt 6):973-82

31. Wang Y, Nickel B, Rutishauser M, Bryce CM, Williams TM, Elkaim G, et al. Movement, resting, and attack behaviors of wild pumas are revealed by triaxial accelerometer measurements. Mov Ecol. 2015;3(1):2-015-0030-0 eCollection 2015.

32. Wilmers CC, Wang Y, Nickel B, Houghtaling P, Shakeri Y, Allen ML, et al. Scale dependent behavioral responses to human development by a large predator, the puma. PLoS One. 2013;8(4):e60590.

33. Chassin PS, Taylor CR, Heglund NC, Seeherman HJ. Locomotion in lions: energetic cost and maximum aerobic capacity. Physiol Zool. 1976:49:1-10.

34. Hoyt DF, Taylor CR. Gait and the energetics of locomotion in horses. Nature. 1981:292(5820):239.

35. Raab JL, Eng P, Waschler RA. Metabolic cost of grade running in dogs. J Appl Physiol. 1976:41(4):532-5.

36. Schmidt-Nielsen K. Animal physiology: adaptation and environment. Cambridge: Cambridge University Press; 1997.

37. Pyke GH. Optimal foraging theory: a critical review. Annu Rev Ecol Syst. 1984;15(1):523-75.

38. Wilson RP, Quintana F, Hobson VJ. Construction of energy landscapes can clarify the movement and distribution of foraging animals. Proc Biol Sci. 2012:279(1730):975-80

39. Burdett CL, Crooks KR, Theobald DM, Wilson KR, Boydston EE, Lyren LM, et al. Interfacing models of wildlife habitat and human development to predict the future distribution of puma habitat. Ecosphere. 2010;1 (1):1-21.

40. Ohmura H, Mukai K, Takahashi T, Aida H, Jones JH. Cardiorespiratory function in thoroughbreds during locomotion on a treadmill at an incline or decline. Am J Vet Res. 2017;78(3):340-9.

41. Taylor CR, Maloiy GM, Weibel ER, Langman VA, Kamau JM, Seeherman HJ, et al. Design of the mammalian respiratory system. III. Scaling maximum aerobic capacity to body mass: wild and domestic mammals. Respir Physiol. 1981:44(1):25-37.

42. Rees WG. Least-cost paths in mountainous terrain. Comput Geosci. 2004; 30(3):203-9.

43. Speakman JR, Król E. Maximal heat dissipation capacity and hyperthermia risk: neglected key factors in the ecology of endotherms. J Anim Ecol. 2010; 79(4):726-46.

44. Taylor, CR, Schmidt- Nielsen, K, Raab, JL. Scaling of energetic cost of running to body size in mammals. Am. J. Physiol. 1970;219(4):1104-1107.

45. Wilson RP, Neate A, Holton MD, Shepard EL, Scantlebury DM, Lambertucci $S A$, et al. Luck in food finding affects individual performance and population trajectories. Curr Biol. 2018;28(23):3871-3877. e5.

46. Minetti AE, Moia C, Roi GS, Susta D, Ferretti G. Energy cost of walking and running at extreme uphill and downhill slopes. J Appl Physiol. 2002;93(3): 1039-46.

47. Bidder OR, Soresina M, Shepard EL, Halsey LG, Quintana F, Gómez-Laich A et al. The need for speed: testing acceleration for estimating animal travel rates in terrestrial dead-reckoning systems. Zoology. 2012;115(1):58-64.

48. Halsey LG. Terrestrial movement energetics: current knowledge and its application to the optimising animal. J Exp Biol. 2016;219(Pt 10):1424-31.

49. Lempidakis E, Wilson RP, Luckman A, Metcalfe RS. What can knowledge of the energy landscape tell us about animal movement trajectories and space use? A case study with humans. J Theor Biol. 2018:457:101-11.

50. Dunford CE, Marks NJ, Wilmers CC, Bryce, CM, Nickel B, Wolfe LL, Scantlebury DM, Williams TM. Puma data, dryad, Dataset, https://doi.org/10. 5061/dryad.4qrfj6q6w 2020.

\section{Publisher's Note}

Springer Nature remains neutral with regard to jurisdictional claims in published maps and institutional affiliations. 